\title{
Progress Toward Future Runway Management
}

\author{
Gary W. Lohr ${ }^{*}$, Sherilyn Brown \\ National Aeronautics and Space Administration, Hampton, Virginia, 23681 \\ Steve Atkins ${ }^{\dagger}$ \\ Mosaic ATM, Inc, Leesburg, Virginia, USA \\ Steve Eisenhawer, Terry Bott \\ Logic Evolved Technologies, Sante Fe, New Mexico, USA \\ And \\ Dou Long, Shahab Hasan* \\ Logistics Management Institute, McLean, Virginia, USA
}

\begin{abstract}
The runway is universally acknowledged as a constraining factor to capacity in the National Airspace System (NAS). It follows that investigation of the effective use of runways, both in terms of selection and assignment, is paramount to the efficiency of future NAS operations. The need to address runway management is not a new idea; however, as the complexities of factors affecting runway selection and usage increase, the need for effective research in this area correspondingly increases. Under the National Aeronautics and Space Administration's Airspace Systems Program, runway management is a key research area. To address a future NAS which promises to be a complex landscape of factors and competing interests among users and operators, effective runway management strategies and capabilities are required. This effort has evolved from an assessment of current practices, an understanding of research activities addressing surface and airspace operations, traffic flow management enhancements, among others. This work has yielded significant progress. Systems analysis work indicates that the value of System Oriented Runway Management tools is significantly increased in the metroplex environment over that of the single airport case. Algorithms have been developed to provide runway configuration recommendations for a single airport with multiple runways. A benefits analysis has been conducted that indicates the SORM benefits include supporting traffic growth, cost reduction as a result of system efficiency, NAS optimization from metroplex operations, fairness in aircraft operations, and rational decision making.
\end{abstract}

\section{Nomenclature}

$\begin{array}{ll}\text { AAR } & =\text { Airport Acceptance Rate } \\ \text { ADR } & =\text { Airport Departure Rate } \\ \text { ASP } & =\text { Airspace Systems Program } \\ \text { ATC } & =\text { Air Traffic Control } \\ \text { ATCT } & =\text { Airport Traffic Control Tower } \\ \text { CADRS } & =\text { Combined Arrival/Departure Runway Scheduling } \\ \text { CDA } & =\text { Continuous Descent Approaches }\end{array}$

\footnotetext{
* Aerospace Engineer, Research Technology Directorate, 15 S. Wright Street, Hampton, VA, Senior Member

${ }^{\dagger}$ Vice President and Principal Analyst, 801 Sycolin Rd. SE, Ste. 306, Leesburg, VA 20175, Senior Member

* Program Director, Investment \& Cost Analysis, 2000 Corporate Ridge, McLean VA 22102, Senior Member
} 


$\begin{array}{ll}\text { ConOps } & =\text { Concept of Operations } \\ \text { DLR } & =\text { Deutsches Zentrum für Luft- und Raumfahrt (German Aerospace Center) } \\ \text { DST } & =\text { Decision support tool } \\ \text { FY } & =\text { Fiscal Year } \\ \text { ILS } & =\text { Instrument Landing System } \\ \text { IMC } & =\text { Instrument meteorological conditions } \\ \text { JPDO } & =\text { Joint Planning and Development Office } \\ \text { LAHSO } & =\text { Land and hold short operations } \\ \text { NAS } & =\text { National Airspace System } \\ \text { NASA } & =\text { National Aeronautics and Space Administration } \\ \text { NextGen } & =\text { Next Generation Air Transportation system } \\ \text { ORD } & =\text { Chicago O'Hare International Airport } \\ \text { RCM } & =\text { Runway Configuration Management } \\ \text { RCP } & =\text { Runway Configuration Plan } \\ \text { Rwy } & =\text { Runway } \\ \text { SORM } & =\text { System Oriented Runway Management } \\ \text { SRCM } & =\text { Strategic Runway Configuration Management } \\ \text { TRACON } & =\text { Terminal Radar Approach Control } \\ \text { TFM } & =\text { Traffic Flow Management } \\ \text { TMI } & =\text { Traffic Management initiative } \\ \text { VHF } & =\text { Very high frequency } \\ \text { TRCM } & =\text { Tactical Runway Configuration Management } \\ \text { VMC } & =\text { Visual meteorological conditions } \\ \text { VOR/DME } & =\text { VHF omnidirectional range/ distance measuring equipment }\end{array}$

\section{Introduction}

The runway is a constraining factor to air traffic operations in the United States National Airspace System (NAS). The Concept of Operations (ConOps) for the Next Generation Air Transportation System (NextGen) states, "runway capacity at the busiest airports is the primary limiting factor in National Airspace System (NAS) operations today...". Because runways are a resource for departures, arrivals, and taxiing aircraft, effective planning of runway usage has significant impact on air traffic operations. The runway, although technically part of the airport surface, can be thought as a separate entity situated at the transition point between surface and airspace domains.

As part of its roadmap for future work through Fiscal Year $2013^{2}$, the Joint Planning and Development Office (JPDO) has identified four research activities that relate to runway management: Integration of Arrival/Departure and Surface Operations, Metroplex Throughput Optimization, Surface Management System, and Implementation of Metroplex Concept. In the creation of a research area to address runway management under the National Aeronautics and Space Administration's (NASA's) Airspace Systems Program (ASP), an assessment of current practices was undertaken to establish a benchmark ${ }^{3}$. Based on this assessment, which included a survey of 15 air traffic control towers (ATCT's) serving high demand airports and five Terminal Radar Approach Controls (TRACONs), it was determined that there was little automation used in the runway management process. Air traffic personnel have done an excellent job in managing runways based on experience and "rules of thumb"; however, the air traffic flow landscape promises to become more complex. Research efforts are underway to improve efficiency in many areas. For example, progress has been made in reducing separation standards based on a better understanding of wake vortex hazards; a rule change was made that reduces required diagonal separation from 2 miles to $1 \frac{1}{2}$ miles for large category aircraft ${ }^{4}$.

Ongoing research to quantify wake hazards under various condition shows promise in eventual re-evaluation of exiting wake vortex separation standards. Continuous Descent Approaches (CDAs) have significant potential to increase efficiency for aircraft arrivals; however, runway assignments assumed for given aircraft may not be the final assignment. Runway management strategies will provide continuous re-evaluation of such assignments and ensure they are consistent with system objectives. Dynamic airspace research is intended to balance airspace allocation with concurrent demand, and will require coordination with runway management strategies. These, as well as other concepts under investigation will require coordination with runway management strategies. 
The initial thrust for the runway management work under the ASP was to address "runway balancing" which is a term used generically with respect to the allocation of traffic across runways. By definition the term suggests parity or equitable distribution of traffic among active runways. Re-evaluation of NAS needs and refinement of research direction resulted in the expansion of the runway management work to consider both arrivals and departures, and to adopt a systemic approach to how runways are used. Hence, the fundamental premise behind the SORM concept is that traffic flow management (TFM) will play an ever-increasing role in the movement of aircraft in the NAS. Further, the selection of runway configurations (a set of runways, usually pre-defined), and the assignment of aircraft to those runways have to support TFM objectives. Research under SORM is based on a two phase approach: single-airport and multiple-airport, or metroplex operations. As significant progress has been made on the former capability, current emphasis is shifting to address runway management in a metroplex environment.

This paper discusses the SORM concept, factors affecting runway configuration selection, runway configuration algorithm development, development of a simulation environment to support algorithm development, and systems analysis.

\section{SORM Concept}

System Oriented Runway Management is composed of two basic capabilities: Runway Configuration Management (RCM), and Combined Arrival/Departure Runway Scheduling (CADRS). Runway Configuration Management is the process of designating active runways, monitoring the active runway configuration for suitability given existing factors, and predicting future configuration changes. Combined Arrival/Departure Runway Scheduling is the process of distributing arrivals and departures across active runways based on local airport and NAS goals.

\section{A. Runway Configuration Management}

Runway Configuration Management is presented as two separate capabilities, Strategic RCM (SRCM) and Tactical RCM (TRCM), that are used in substantially different ways and at unequal levels by those involved in the traffic flow process, and they involve operations at different time scales. Traffic Flow Management requires an estimate of airport capacity that may be used in planning traffic management initiatives (TMI) several hours in advance. However, since the TMIs depend on the capacities, SRCM will plan airport capacities in concert with TFM planning the TMIs. Controllers and traffic managers at and near the airport require a runway configuration plan over the next hour or sooner. The significant difference in the uncertainty characteristics of these time scales is expected to result in different algorithms being used, further warranting the separation. TRCM will plan the airport configuration to best satisfy demand over the next hour. In the far-term, as uncertainty is reduced through other technologies, strategic and tactical RCM may merge. Note that in this document, the use of RCM refers to a combination of the TRCM and SRCM capabilities.

\section{B. Combined Arrival/Departure Runway Scheduling}

CADRS will provide a tool for coordinating runway planning, runway assignments and sequencing or scheduling - so that all of the relevant factors are considered, including airspace, airport surface, and TFM. It is assumed that default runway assignments will continue to be issued; however, based on factors such as surface congestion and greater accommodation of user preferences, runway assignements may be altered. Such changes to runway assignments may have to be issued in a timely manner.

\section{Approach}

\section{A. Factors}

Many factors affect the selection of an airport's active runway configuration. Major considerations at high traffic volume facilities include:

Winds

Traffic demand

Terminal traffic flow
Environmental considerations

User requirements

User preferences 
Airport Operator requirements

Availability of Land and Hold Short Operations

(LAHSO)

Spacing between parallel runways

Airspace restrictions
Surface restrictions

Dedicated vs. dual use runways

Balancing by aircraft type

Staffing issues

Metroplex considerations

Most of these factors can be categorized as: constraints, preferences, procedures, and "other". Constraints are limitations on the ability to exercise discretion regarding runway management choices, e.g., winds above acceptable tailwind thresholds, environmental (e.g., noise), surface traffic restrictions, and user requirements (e.g., runway length). Preferences are essentially requests that would be accommodated as conditions permit. Procedural considerations are those would have to be factored into runway management decisions in order to realize end state efficiency objectives, an example being the use of LAHSO procedures.

The selection of a runway configuration is first and foremost based on safety; therefore, winds and other weather related phenomenon are the primary considerations. Wind velocity is the primary driver when selecting a runway configuration. When maximum tailwind limits ${ }^{4,5}$ are exceeded, that runway cannot be assigned. Procedures that improve efficiency are considered in runway configuration selection, for example, "are there sufficient weather minimums to permit reduce departure separation based on divergent headings?", or "are LAHSO operations authorized based on prescribed criteria?"

\section{B. Target Airports}

The SORM -capabilities under development are intended to be beneficial at essentially all airports; however, benefits analyses suggest that the benefits increase with increased traffic density. Clearly, many airports will not require such capabilities.

For the development work under the SORM effort, operational environments were selected that are intended to fully exercise the envisioned capabilities. For the single airport case, John F. Kennedy International Airport (KJFK) was selected. The airport layout at KJFK, along with supporting air traffic procedures, incorporates the characteristics of those found at high traffic volume airports throughout the country. The New York terminal area was selected for the development of capabilities needed to support metroplex runway management. Characterized by complex and congested airspace, the New York area has been the subject of several efforts to quantify operations and identify constraints. Current and future research will leverage on these efforts. Several other airports, apart from KJFK, were the subject of analysis in the algorithm development process. This was to ensure that the broadest exercise of SORM capabilities was conducted.

\section{System Analysis}

System analysis studies for SORM were carried out in two phases: single-airport and metroplex. In Phase One, system models for CADRS and RCM at a single airport were developed. The approach taken was to model runway operations in considerable detail at specific large hub airports where SORM-based decision support tools (DST) would be expected to offer significant advantages over current runway management techniques. The system analysis for CADRS at Los Angeles International Airport (KLAX) is reported in Reference 6. An RCM DST can operate at either the single airport or metroplex-level. Airport-level RCM (Airport-RCM) is appropriate when the airport operations have little or no interaction with the operations at other airports. For the Phase One analysis the airport modeled was Chicago O'Hare International Airport (KORD) ${ }^{*}$. Analyses performed with the airport-level system model for KORD showed that an RCM DST had significant potential to improve the decision process by selecting Runway Configuration Plans (RCPs) consistently to maximize throughput and by minimizing delays associated with the RCP transitions (Reference 7,8). Inter-airport interactions of interest for RCM arise when selection of the RCP at one airport affects the Airport Acceptance Rate (AAR) or Airport Departure Rate (ADR) associated with the RCPs at one or more nearby airports. These interactions are an effect of the density of arrival and departure operations in the terminal airspace that cannot be eliminated by ATC procedures alone. The

\footnotetext{
${ }^{*}$ A single interaction between KORD and Midway International Airport was considered separately as part of the Phase One analysis.
} 
complexity of the airspace near closely-spaced airports introduces effects that must be accounted for by the RCM DST. The granularity of the airspace interactions among metroplex airports can be quite fine and occurs at the individual runway pair level. Capacities on one or both runway ends will be reduced for any combination of RCPs at the two airports where this runway pair appears. Figure 1 shows the constrained airspace between KJFK and La Guardia Airport (KLGA) in New York. In addition to pair-wise capacity reductions, certain combinations of runways cannot be used together because of aircraft separation requirements. For example, when KJFK uses the Instrument Landing System (ILS) approach to Rwy 13L, KLGA cannot use arrivals to Rwy 04, Rwy 22 or the favored Expressway 31 visual approach to Rwy 31.

Additional metroplex effects arise from the fact that forecast dynamic conditions (e.g., wind direction and speed, visibility and ceiling, runway condition) and operational state (e.g., arrival or departure bank, LAHSO, ground stops in effect) cannot be assumed to change simultaneously nor in an identical way at the dependent airports. The winds, visibilities, and ceilings at proximate airports can be significantly different and improve or degrade at different times and rates. The relative importance of these factors can be expected to be strongly dependent on the metroplex under consideration.

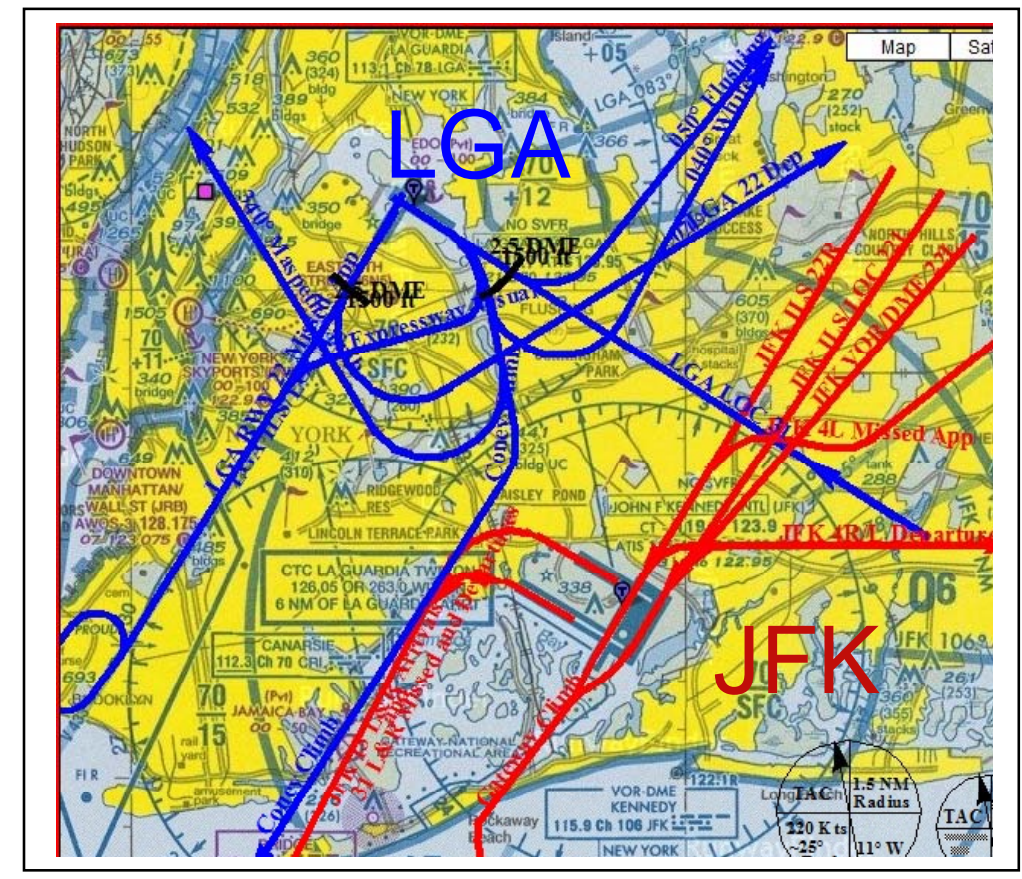

Figure 1. KJFK and KLGA runway interactions

The development of a system model for Runway Configuration Management for a metroplex (metroplex-RCM) (Reference 8) builds upon the Phase One RCM system model for a single airport. The emphasis of the analysis was on understanding what new, additional input variables are needed for metroplex-RCM. The approach was to modify and extend the airport-level RCM system model to account for these inputs as dependent metroplex models for New York, the San Francisco Bay Area and Los Angeles were constructed. Personnel from the three TRACONs (N90, $\mathrm{NCT}$, and SCT) were interviewed to understand the nature of the constraints on RCM and to determine which airports to model. At New York, four airports were modeled: KJFK, KLGA, Newark Liberty International (KEWR) and Teterboro (KTEB). For the Bay Area, San Francisco International Airport (KSFO), Metropolitan Oakland International (KOAK) and San Jose International (KSJC) were modeled. For Los Angeles, Los Angeles International (KLAX), Bob Hope - Burbank (KBUR) and Van Nuys (KVNY) were included. Although other airports may be proximate to the modeled airports, the effect on operations is negligible as a result of ATC procedures, the structure of the airspace, or the types of aircraft being handled.

Airports in a metroplex can be weakly or strongly coupled. For the case where the coupling is weak, an AirportRCM analysis using the Phase One methodology was deemed appropriate. In the strongly coupled case, the approach shown in Figure 2 was developed and can be summarized briefly as follows. The airport-level model was used in a first iteration to rank order the RCPs at each airport assuming the airports are independent. That is, the 
selection of an RCP is not affected by RCP decision-making at the other metroplex airports. These rankings were based on an aggregate RCP attractiveness metric computed using a model very similar to the single airport model. Airport level attractiveness is an aggregation of factors including arrival and departure capacity-demand ratios for three aircraft weight classes and controller and aircrew workloads. They are used to down select plans for further consideration. The down-selected plans were combined into a set of $\mathrm{N}$-tuples where $\mathrm{N}$ is the number of airports for which RCP changes are under consideration. The runway capacities for each RCP in the N-tuple are adjusted for metroplex effects and the airport-level aggregate metric was re-computed with updated inputs. Finally, the set of Ntuples with the updated attractiveness metrics for each RCP were input to a second ranking model to generate a metroplex-level attractiveness metric for each N-tuple. The rank ordering of the RCP N-tuples is one of the two primary outputs of a RCM DST.

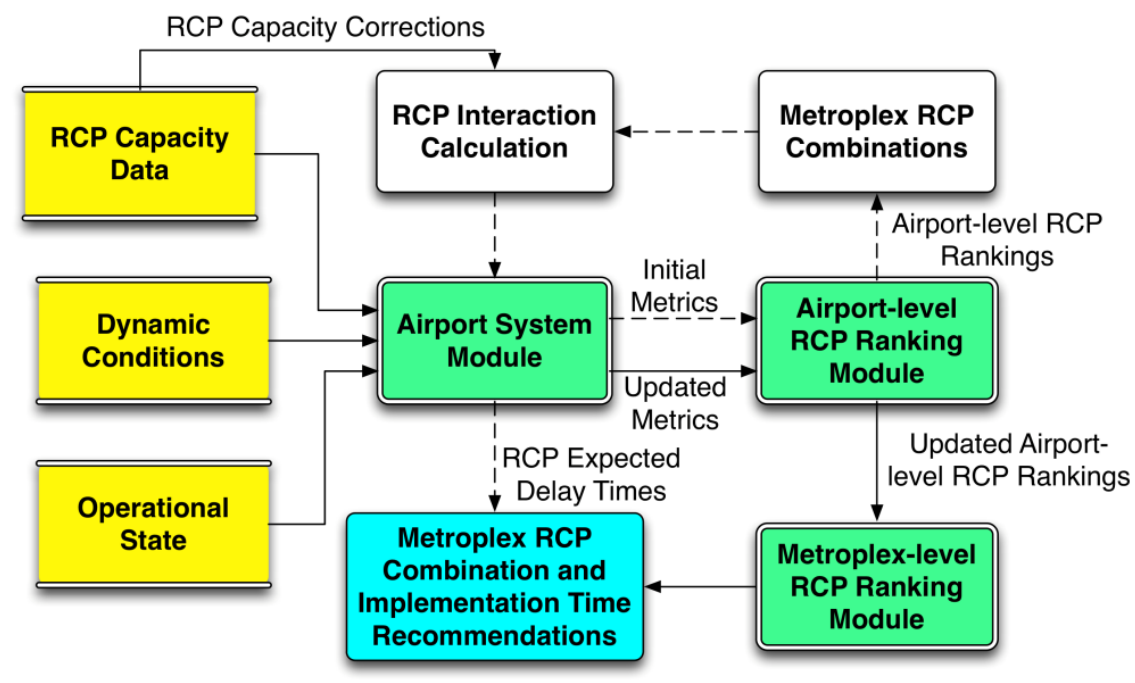

Figure 2. Overview of metroplex-RCM system model analysis process

A range of cases was analyzed for all three metroplexes. Figure 3 shows the airport-level RCP rankings for KJFK for a high-wind case, with instrument meteorological conditions and runway 22L closed. The effect of closing Rwy $22 \mathrm{~L}$ is to force the use of RCP ILS 13L $\mid 13 \mathrm{R}^{*}$. This is the only RCP with a high value of the ranking metric called 'attractiveness' $(A): A$ is the output of the ranking module.

\footnotetext{
* Runway designators to the left of the vertical bar are arrival runways; those to the right are departure runways. Two runways used for the same operation are separated by an ampersand. A dual use runway will appear on both sides of the vertical bar.
} 


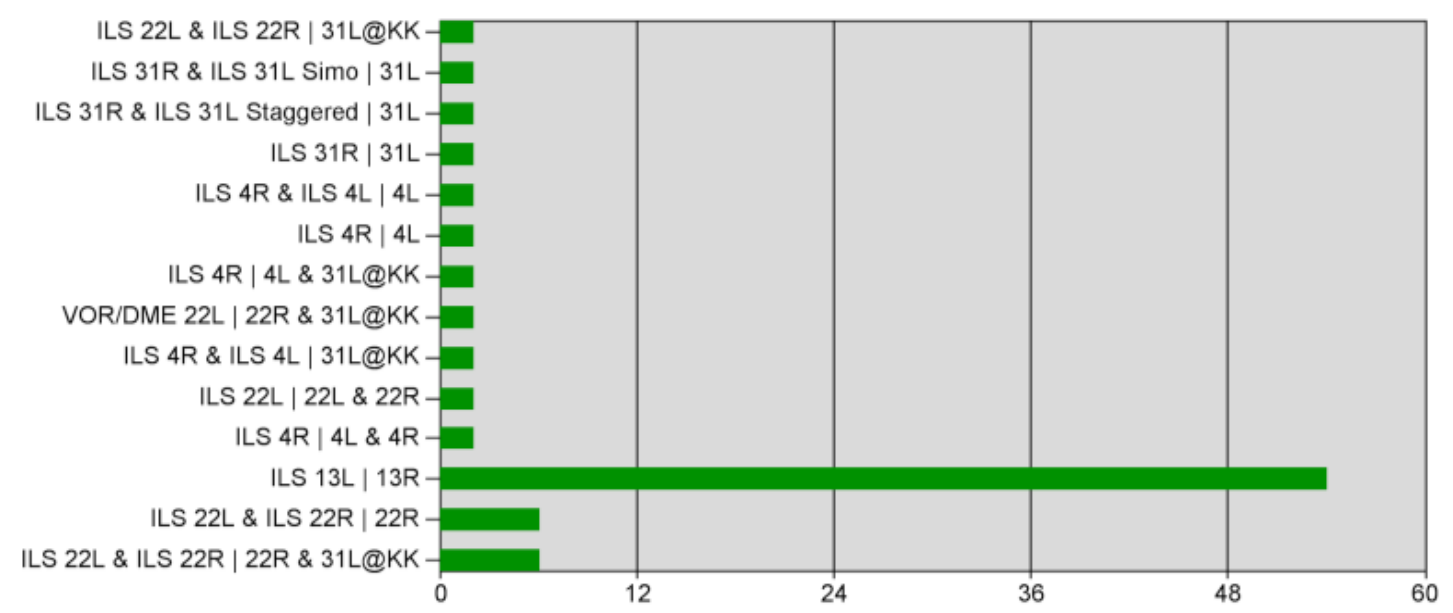

Figure 3. KJFK RCP Attractiveness

Candidate RCPs are found based on the $A$ metric and taking into account ATC preferences, and then are assembled into N-tuples ${ }^{*}$. Each N-tuple is evaluated for pair-wise RCP interactions. If a non-zero interaction is present, then the effect on runway capacity is calculated. Incompatible RCP combinations that are not possible because of airspace constraints as noted above are discarded. Figure 4 shows the metroplex-level $A$ scores for the candidate N-tuples. If no incompatibles exist, there would be 16 candidates. However because the KJFK RCP (denoted as J7) with Rwy 13L conflicts with arrivals on KLGA Rwy 22 in the RCP (designated as L29) this N-tuple does not appear.

The case studies with the three metroplex models indicated that the New York metroplex, as expected, is by far the most complex for metroplex-RCM. The number of airports combined with the large number of RCP pairs that interact means that a large solution space must be examined. The presence of incompatible RCP pairs across multiple airports, e.g. KLGA-KJFK, KLGA-Teterboro (KTEB) is not seen for the other two metroplexes. In the Bay Area, the use of West Plan and Southeast Plans at KSFO simplifies RCM. RCP selection at KOAK is basically forced to be in phase with KSFO. The interaction of KSJC with KSFO in one situation is strong in the sense that the $\mathrm{RCP}$ at KSFO using Simultaneous Offset Instrument Approaches (SOIA) is eliminated by an airspace constraint. However this only affects arrival capacity; the actual arrival runways do not change. Modeling the Los Angeles metroplex for RCM did not introduce any new complications. The nature and strength of inter-airport interactions across the metroplexes provides valuable information about where airport-RCM versus metroplex-RCM DSTs would be needed.

Future research topics suggested by this study include the sensitivity of the use case results to the methods used to select candidate RCPs and the potential benefits of fine tuning the ranking models to individual airports. Overall, the use of the models to learn more about the RCM/CADRS interface and to explore approaches to Collaborative Decision Making is also recommended.

\footnotetext{
${ }^{*}$ For New York there are four airports so $\mathrm{N}=4$
} 


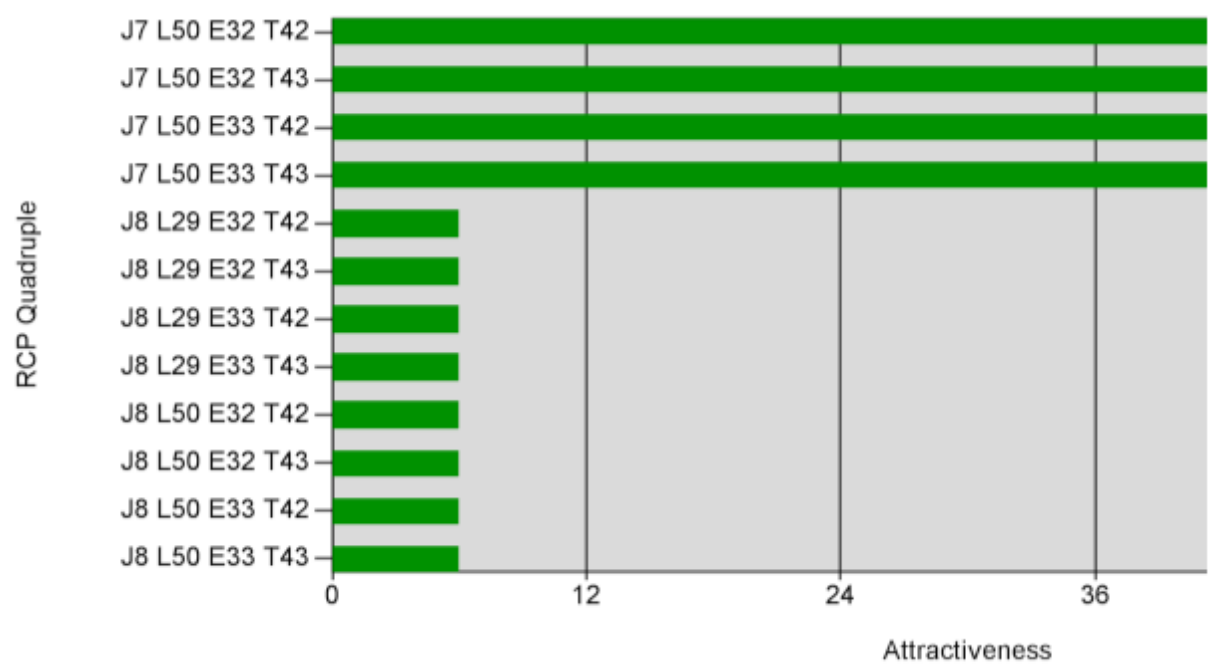

Figure 4. New York RCP N-Tuple attractiveness

\section{Algorithms}

The algorithms are the core of the TRCM capability. Building on the work of Weld, Duarte, and Kincaid (Reference 10), an instance of the TRCM problem is defined by a set of flights, F, representing all arrivals and departures predicted to operate at the airport or metroplex from the current time through a specified modeling horizon. TRCM is defined on a continuous time scale and it is assumed that the current time has been normalized to zero. Parameters $h_{f}$ and $h_{p}$ are the freeze and planning horizon, respectively, and both are assumed to be greater than zero. No new configuration changes can be scheduled prior to time $h_{f}$ or after time $h_{p}$. In addition, any configuration changes scheduled between the current time and $h_{f}$ cannot be canceled. In general, $h_{f}$ and $h_{p}$ may be different for each airport configuration element, such as runway configuration and runway assignment policy.

It is assumed that a maximum number, $M$, of configuration changes are allowed between $h_{f}$ and $h_{p}$. The set of configurations is indexed by $\mathrm{k} \in\{1,2, \ldots, \mathrm{K}\}$. There are two sets of decision variables defined for each configuration k. For each $1 \leq \mathrm{m} \leq \mathrm{M}, \Delta_{\mathrm{mk}}$ is a binary decision variable equal to 1 if the $\mathrm{m}$ 'th configuration change is to configuration $\mathrm{k}$ and equal to zero otherwise. $\tau_{\mathrm{mk}}$ is a continuous variable that is equal to the time of the m'th configuration change when $\Delta_{\mathrm{mk}}=1$ and equal to zero otherwise. We define the matrices $\Delta=\left[\Delta_{\mathrm{mk}}\right]$, which contains all binary decision variables, and $\tau=\left[\tau_{\mathrm{mk}}\right]$, which contains all configuration change time decision variables.

The information state of the airport at the current time is designated as $\pi$. The information state includes weather forecasts, scheduled configuration changes up to the freeze horizon, scheduled traffic management initiatives, runway and taxiway closures, and any other information affecting the operation of the airport.

$$
\begin{aligned}
\operatorname{minimize} C(F, \pi, \tau) & \\
\text { s.t. } h_{f} \Delta_{m k} \leq \tau_{m k} & \leq h_{p} \Delta_{m k} \forall m, k \\
\tau_{m^{\prime} k^{\prime}}-\tau_{m k} & \geq B_{k k^{\prime}}-\left(1-\Delta_{m^{\prime} k^{\prime}} \Delta_{m k}\right) \bar{B} \forall m, k, m^{\prime}>m, k^{\prime} \neq k \\
\sum_{m k}^{k} \Delta_{m} & \leq 1 \forall m \\
\sum_{k} \Delta_{m k} & \geq \sum_{k} \Delta_{m+1, k} \forall m \\
\Delta_{m k}+\Delta_{m+1, k} & \leq 1 \forall m, k \\
\tau & \in A(\pi) \\
\Delta_{m k} & \in\{0,1\} \forall m, k
\end{aligned}
$$


The objective function $\mathrm{C}(\mathrm{F}, \pi, \tau)$ is a generalized expected cost function over the set of flights $\mathrm{F}$ given information state $\pi$ and configuration change time decision variable $\tau$. Potential cost metrics include flight delays, flight and taxi times, and fuel burn. The first constraint ensures that each configuration change time is nonzero if and only if the corresponding binary decision variable is set to 1 and any nonzero configuration change time falls between the freeze horizon and the planning horizon. The second constraint requires a separation of $\mathrm{B}_{\mathrm{kk}}$ ' between configuration changes to configuration $\mathrm{k}$ and to configuration $\mathrm{k}^{\prime}$. This allows different configuration pairs to have different restrictions on how frequently changes may be made. For example, the direction in which the runways are used may be changed infrequently, while adding or removing a runway operating in the same direction can be done more frequently. Constant $\overline{\mathrm{B}}$ is defined large enough so that this constraint is inactive when either $\Delta_{\mathrm{m}} \mathrm{k}^{\prime}$ or $\Delta_{\mathrm{mk}}$ is zero. The third constraint ensures that at most one configuration is chosen for each configuration change. The fourth constraint condenses the configuration changes by allowing a $(m+1)$ 'th configuration change only if an m'th configuration change has been selected. The fifth constraint does not allow consecutive configuration changes to the same configuration. We define $\mathrm{A}(\pi)$ as the feasible space for configuration change times based on the current information state. Thus, the sixth constraint allows for airport- and scenario-specific constraints such as noise abatement procedures or resource usage restrictions. The final constraint defines the $\Delta_{\mathrm{mk}}$ to be binary variables. The binary variables and the non-linearity of the second constraint in the above formulation imply that the state space is non-convex. In addition, the expected cost function $C(F, \pi, \tau)$ will be difficult or impossible to compute directly due to the complexity of predicting flight operations. Therefore, a solution heuristic is replaced that makes a few simplifying assumptions

First, the cost function $C(F, \pi, \tau)$ is replaced with a simplified cost function $\tilde{C}(F, \pi, \tau)$ that can be computed via fast-time simulation. Any such function can be plugged into the solution heuristic. A fast-time simulation model, however, will be dependent on deterministic gate and fix times for arrivals and departures, respectively. In reality, there is a significant amount of error in flight time predictions. This uncertainty is accounted for by adding a Monte Carlo sampling scheme to the model. Error distributions are defined for the pushback times of departures and the fix crossing time of arrivals. A sample flight list is created by drawing a sample from the appropriate distribution for each flight in flight list $\mathrm{F}$ and applying that error to the flight's gate or fix time. Let $\mathrm{F}_{\mathrm{i}}$ be the $\mathrm{i}$ 'th sample flight list. If $\mathrm{N}$ samples are drawn, then the objective function in the TRCM problem formulation is replaced with

$$
\text { minimize } \frac{1}{N} \sum_{i=1}^{N} \tilde{C}\left(F_{i}, \pi, \tau\right) .
$$

The second simplification of the heuristic is a discretization of the state space. It is assumed that configuration changes can only be scheduled at predetermined times through the planning horizon and that configuration changes will only be planned at five-minute intervals. Future work will study removing this restriction, possibly through a second stage of the algorithm. To simplify implementation, it is also assumed that the planning horizon falls on such an interval. Given $\pi$, the possible configuration list may be reduced using heuristics. For example, configurations only allowed under visual meteorological conditions (VMC) do not need to be considered when the weather will be instrument meteorological conditions (IMC).

The above simplifications allow for a basic enumerative search to be used to solve the TRCM problem. The TRCM search heuristic is a recursive search algorithm. Each recursive call to the algorithm adds a new configuration change to the end of the list of configuration changes. The algorithm maintains a single best global solution $\left(\Delta^{*}, \tau^{*}\right)$ at all points in the algorithm and at all depths of the recursion. The algorithm is seeded with the solution $\Delta=\tau=[0]$ for all $\mathrm{m}$ and $\mathrm{k}$, which corresponds to no new configuration changes, and with the current best objective function value $\mathrm{z}^{*}$ equal to infinity.

$T R C M \_\operatorname{Search}\left(\Delta, \tau, \Delta^{*}, \tau^{*}, z^{*}\right)$

Evaluate $z=\frac{1}{N} \sum_{i=1}^{N} \tilde{C}\left(F_{i}, \pi, \tau\right)$.

If $z \leq z^{*}$ then set $\Delta^{*}=\Delta, \tau^{*}=\tau$, and $z^{*}=z$.

Let $m$ be the smallest value for which $\sum_{k} \Delta_{m k}=0$. If no such $m$ exists then return $\left(\Delta^{*}, \tau^{*}, z^{*}\right)$ and exit.

If $m=0$ then set $t=h_{f}$. Else set $t$ to be the first interval time after the $(m-1)^{\text {th }}$ configuration change time.

For each configuration $k$ :

Set $\Delta_{m k}=1$.

For each interval time $t^{\prime}$ from $t$ to $h_{p}$ :

Set $\tau_{m k}=t^{\prime}$. 
If $\tau$ is a feasible set of configuration change times then recursively call TRCM_Search $\left(\Delta, \tau, \Delta^{*}, \tau^{*}, z^{*}\right)$ and set $\left(\Delta^{*}, \tau^{*}, z^{*}\right)$ to the returned values.

Set $\Delta_{m k}=\tau_{m k}=0$.

$\operatorname{Return}\left(\Delta^{*}, \tau^{*}, z^{*}\right)$ and exit.

The above algorithm will return the optimal set of configuration changes over the planning period relative to objective function $\frac{1}{\mathrm{~N}} \sum_{\mathrm{i}=1}^{\mathrm{N}} \tilde{\mathrm{C}}\left(\mathrm{F}_{\mathrm{i}}, \pi, \tau\right)$ subject to the constraints of the original TRCM problem over the discretized state space. In operation, the algorithm runs at a periodic rate such that there is significant overlap in the planning windows from one iteration to the next, each iteration beginning with the prior best solution.

An example of the system perspective of RCM is how limited capacity at a departure fix is handled. If the airport is not able to depart a large number of aircraft due to a downstream restriction, then there is no need to select a runway configuration with a high departure capacity. Instead, the runway configuration may be selected for higher efficiency (e.g., shorter taxi paths and more direct airborne routes) or higher arrival capacity if sufficient arrival demand exists. However, RCM does not only consider the overall capacities and demands but how specific resources will be used. For example, if in a particular runway configuration one departure runway would only be used by flights to specific departure fixes that have limited capacity (due to TFM restrictions) or are closed due to weather, or for which little demand exists, then RCM will recognize that the runway configuration does not fully utilize that runway and results in higher overall cost than other runway configurations. There may be very high demand to the closed or capacity constrained departure fixes, but since that demand cannot depart, RCM will not select the runway configuration simply based on the demand but rather based on the achievable operations considering demand, runway capacity, and other capacity restrictions.

Additional information regarding the development and evaluation of the TRCM algorithm can be found in Reference 11.

\section{E. Metroplex Simulation Environment}

A Metroplex Simulation Environment (MSE) was developed to provide a Java environment for evaluation of air traffic algorithms within a metroplex region, providing many capabilities that would be common to most simulations, along with an intuitive application programming interface (airportI) to access and extend those capabilities. The simulation environment allows researchers to focus on their specific research questions without the need to implement common functionality. The architecture Figure 5 includes an intuitive object-oriented design with interfaces for many of the processing components. Planners, models of the real world, and aircraft models are all separate components. This design approach allows researchers to extend or replace individual components of the platform. These customized components can implement logic and data management that are unique to a researcher's needs while still interoperating correctly with the rest of the platform. In this way, researchers can leverage and extend the platform to meet their objectives with minimal effort. Multiple configurations of each simulation may be run without recompiling any code by specifying parameters in XML files which are easy to read and edit. The parameterized configuration allows researchers to run single-pass or Monte Carlo style simulations and calculate metrics based on configuration file parameters.

The MSE enables surface and airborne simulations covering multiple airports. To support these simulations, MSE includes detailed aviation data describing airports, runways, taxiways, spots, gates, and fixes. These data are provided through a simple XML "adaptation" file format. Also through XML files, researchers can define the set of flights to simulate, with arrival and departure airports, flight routes, and arrival and departure times for each flight.

By default, MSE simulates flights through an iterative cycle of planning and simulation. The planning phase of the cycle uses the research algorithm to compute, for example, optimized flight routes and scheduled times at waypoints. The simulation phase validates the robustness of the optimized plan using a modeled representation of real-world conditions. MSE supports aircraft models which can be used in planning and simulation to model aircraft dynamics to varying levels of fidelity. 


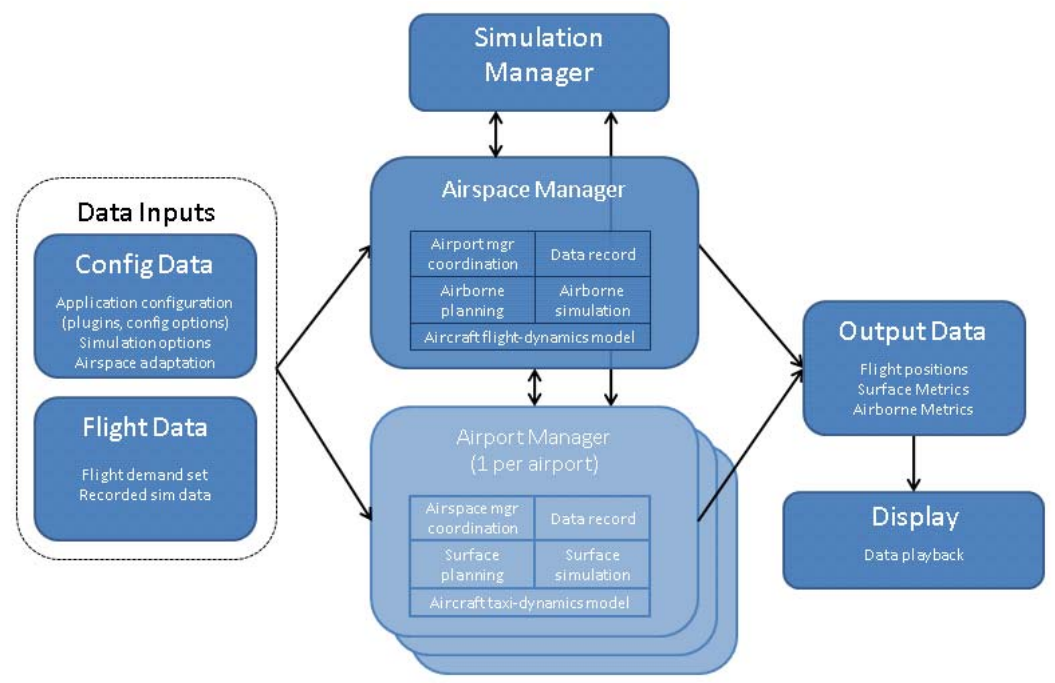

Figure 5. Main Classes of the Metroplex Simulation Environment

\section{F. Fast Time Simulations}

\section{Runway Usage Example}

The TRCM algorithms have been used to investigate potential benefit during the morning traffic period at Memphis International Airport (KMEM). Refer to Figure 6 for the following discussion. Frequently, KMEM operates in one of two equivalent runway configurations in which arrivals land on runways $18 \mathrm{R} / 36 \mathrm{~L}$ and $18 \mathrm{~L} / 36 \mathrm{R}$ and departures take off from runways $18 \mathrm{C} / 36 \mathrm{C}$ and $18 \mathrm{R} / 36 \mathrm{~L}$. Selection of the North Flow or South Flow configuration depends on the wind and traffic characteristics. KMEM serves both a large cargo operation and a passenger operation with separate ramp areas. Which ramp area the majority of the traffic is taxiing from/to is considered when selecting the runway configuration to reduce taxi distances.

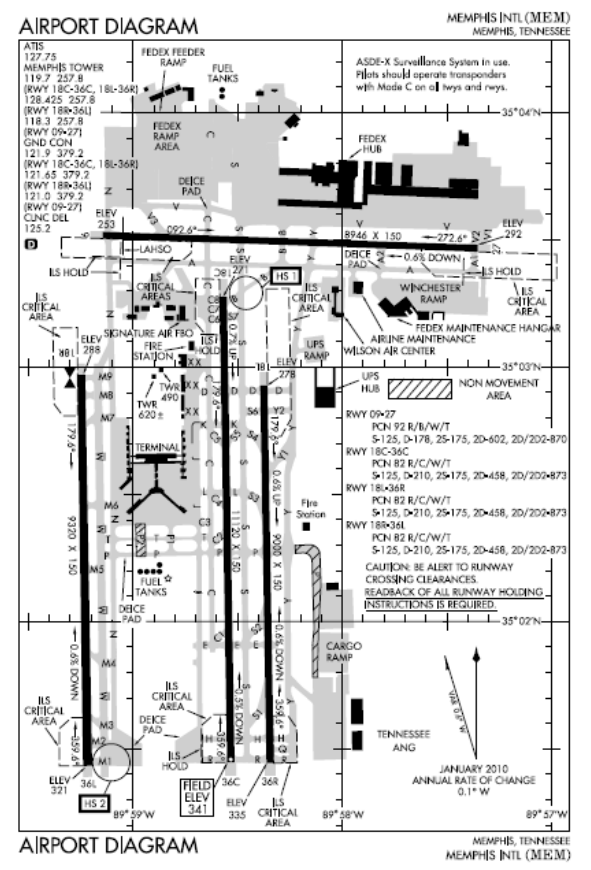

Figure 6. Airport diagram of Memphis International Airport (KMEM) 
To avoid departures crossing in the air, KMEM uses a rigid departure runway assignment rule based on the flight's departure fix. All flights headed to the West are assigned to runway $18 \mathrm{R} / 36 \mathrm{~L}$, while all departures headed to the east are assigned to runway $18 \mathrm{~L} / 36 \mathrm{R}$. Most mornings, between $1300 \mathrm{Z}$ and $1500 \mathrm{Z}$, a cluster of flights depart to the west, which requires them to be assigned to runway $18 \mathrm{R} / 36 \mathrm{~L}$. This departure push overlaps a period of steady arrivals.

Procedures at KMEM allow the TRACON to assign arrivals to either arrival runway. Currently, most TRACON controllers do not consider the departure traffic when assigning arrival runways. To minimize flight time and their own workload, controllers assign arrival flights to the runway closest to the flight's arrival fix. Sometimes during light traffic, controllers will consider where the aircraft will park on the airport.

During this period of time, many of the arrivals are from the west and are assigned to runway $18 \mathrm{R} / 36 \mathrm{~L}$. The arrivals are given priority and the departures form a long queue at $18 \mathrm{R} / 36 \mathrm{~L}$ waiting for infrequent, random gaps in the arrivals sufficient to fit a departure, while runways $18 \mathrm{~L} / 36 \mathrm{R}$ and $18 \mathrm{C} / 36 \mathrm{C}$ are underutilized.

When KMEM is in one of these runway configurations, TRCM advises how the arrival runways should be used. Three runway usage policies are defined: (1) assign arrivals to the runway closest to their arrival fixes, (2) assign arrivals to the runway that minimizes their combined flight and taxi times, and use $18 \mathrm{R} / 36 \mathrm{~L}$ as an overflow arrival runway only after $18 \mathrm{~L} / 36 \mathrm{R}$ is full. While not explicitly defined in the KMEM Standard Operating Procedures (SOPs), the third policy is similar to how KJFK operates- KJFK identifies a primary arrival runway and an overflow arrival runway in each of its two arrival runway configurations. Note that the second policy requires TRCM to model each flight all the way to its parking gate for each possible runway assignment to determine which runway to select. The third policy requires TRCM to model all of the traffic to identify when delays would start to occur on the primary runway such that the overflow runway should begin to be used. The TRCM output specifies which policy should be used and the period of time for which it should be used, the format of which is consistent with current controller decisions. No procedural changes are required.

Tactical Runway Configuration Management was tested using 62 flights that landed or departed KMEM during 1400-1530Z on September 9, 2010. Thirty of the 43 departures were headed west; 10 of 19 arrivals approached from the west. Airborne and surface surveillance data were used to determine the actual controller policy, which was "assign arrival runway based on direction of flight" for the entire time period. TRCM advised using 18L as the primary arrival runway for the entire time period, sending arrivals to $18 \mathrm{R}$ only if $18 \mathrm{~L}$ was being fully used. The TRCM and actual controller runway usage policies were simulated and metrics compared. The simulation considered flying time, runway delay, and taxi time. The TRCM-selected policy reduced the total delay for arrivals and departures to 22.6 minutes, from 45.0 minutes under the controller's policy. Under the TRCM policy, the arrivals experienced slightly more delay than under the controller's actual policy due to a longer flying distance and slight runway delay. However, departures experienced substantially smaller delays on average. While only for a single traffic sample, this example demonstrates the significant benefit possible with an airport configuration management tool.

\section{Runway Assignment Example}

Orlando International Airport (KMCO) has four parallel runways oriented north-south (Figure 7). From west to east, they are $36 \mathrm{~L} / 18 \mathrm{R}, 36 \mathrm{R} / 18 \mathrm{~L}, 35 \mathrm{~L} / 17 \mathrm{R}$, and $35 \mathrm{R} / 17 \mathrm{~L}$. The terminals are between the $36 / 18$ pair and the $35 / 17$ pair and consist of four separate terminal buildings. In South operation, arrivals use the outer runways 36L and 35R, and departures use the inner runways $36 \mathrm{R}$ and $35 \mathrm{~L}$. In North operation, during "severe clear" weather, arrivals land on runways $36 \mathrm{~L}$ and $35 \mathrm{R}$; departures take off from $36 \mathrm{R}$ and $35 \mathrm{~L}$.

MCO operates in two distinct modes. During heavy traffic, departures are assigned to runways based on direction of flight to avoid airborne conflicts and no coordination is required between the two departure runways. This mode is called "taxi for direction [of flight]." During light traffic, the "taxi for convenience" mode allows departures to be assigned to the departure runway closest to the aircraft's parking gate regardless of the direction of flight. In this mode, the local controllers must coordinate releasing aircraft from the two runways to avoid conflicts in the air, and comply with the wake vortex requirements, because the flight paths may cross or merge. The delay at the runway to implement this coordination is small and since traffic is light, departures queues do not accumulate. The SOPs indicate that the supervisor or controller in charge should select which procedure to use. As traffic level increases, there is a cost to using "taxi for convenience" because the small runway delays required to coordinate the runways begin to also delay subsequent flights as a queue forms. However, controllers often switch to "taxi for direction" well before the true efficiency crossover point. Some controllers prefer taxi for direction at all times to reduce their workload. Some controllers use "taxi for convenience" too much, causing flights to be delayed more 
than they would under the "taxi for direction" procedure. TRCM can advise when each runway assignment procedure should be used.

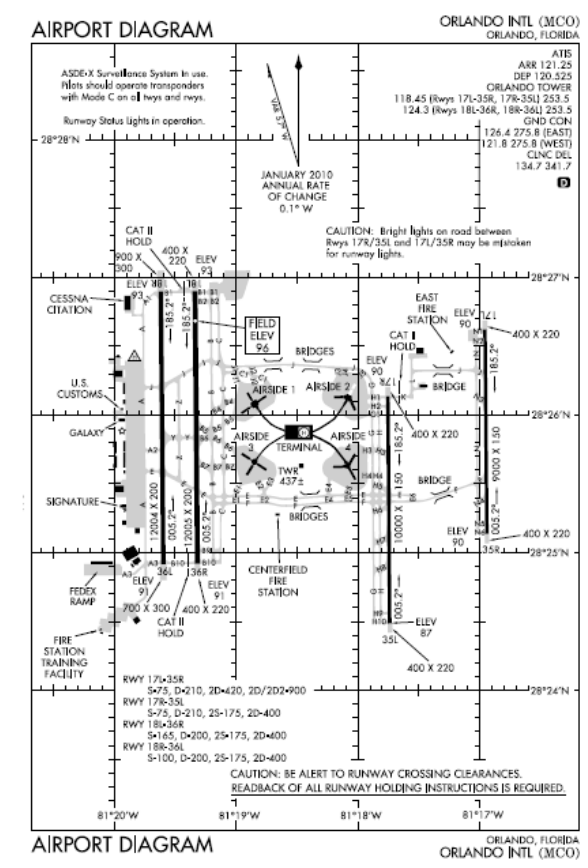

Figure 7. Airport diagram of Orlando International Airport (MCO)

Table 1 shows the taxi distance from each terminal to each departure runway. Assuming a nominal taxi speed of 15 knots, each $1000 \mathrm{ft}$. of taxi takes about 45 seconds. Therefore, runway $35 \mathrm{~L}$ is more than seven minutes farther than runway 36R from Terminal 1 . The difference in flight distance is relatively small in terms of time. The departure runways are separated by $8500 \mathrm{ft}$., which is only about 30 seconds of flying time.

Thirty-eight departures from $1055 \mathrm{Z}$ to $1155 \mathrm{Z}$ on October 13, 2010 were studied. Eleven of 17 flights from the west terminals and 10 of 21 flights from the east terminals departed to the west. KMCO operated in the North-flow configuration with departures on $36 \mathrm{R}$ and $35 \mathrm{~L}$. Based on analysis of airport surface surveillance data, the actual operations were "taxi for direction" throughout the time period. TRCM considered the two runway assignment policies, including the possibility of changing the policy during the time period. TRCM advised that "taxi for convenience" should be used for the entire hour. The policies used historically and advised by TRCM were simulated and metrics compared.

Table 1. Approximate Taxi Distances from Ramps to Departure Runways at KMCO

\begin{tabular}{|l|l|l|}
\hline & Runway 36R & Runway 35L \\
\hline Ramp 1 (north-west) & $8300 \mathrm{ft}$. & $18,200 \mathrm{ft}$. \\
\hline Ramp 2 (north-east) & $14,600 \mathrm{ft}$. & $10,200 \mathrm{ft}$. \\
\hline Ramp 3 (south-west) & $4500 \mathrm{ft}$. & $12,100 \mathrm{ft}$. \\
\hline Ramp 4 (south-east) & $9500 \mathrm{ft}$. & $7100 \mathrm{ft}$. \\
\hline
\end{tabular}

Table 2 summarizes the simulation results. "Taxi for convenience" results in slightly longer runway delays due to the need wait for traffic on the other runway that will cross or require merging. However, "taxi for convenience" produces a significantly smaller delay overall. This delay reduction comes primarily from shorter taxi times since the flight time to the fix from each runway is nearly the same. This example illustrates the potential of TRCM to 
provide significant benefit within existing procedures. Future work will expand TRCM to consider fuel burn and other metrics in addition to time.

Table 2. TRCM Results at KMCO

\begin{tabular}{|l|l|l|} 
& $\begin{array}{l}\text { Taxi for Direction (Actual } \\
\text { Controller Decision) }\end{array}$ & $\begin{array}{l}\text { Taxi for Convenience } \\
\text { (TRCM Output) }\end{array}$ \\
\hline Total Delay & $41.4 \mathrm{~min}$. & $16.5 \mathrm{~min}$. \\
\hline Average (per flight) Runway Delay & $24 \mathrm{sec}$. & $26 \mathrm{sec}$. \\
\hline
\end{tabular}

\section{Runway Configuration Examples}

TRCM is also capable of planning the runway configuration and when to change it. To illustrate, a one-hour period of traffic from KJFK (Figure 8) on March 19, 2009 was studied. Forty departures and 24 arrivals operated during the time period from 2:30 to 3:30 PM local time, which contained a wind shift at 3:00 PM that exceeded the tailwind threshold for runways $22 \mathrm{~L}$ and $22 \mathrm{R}$.

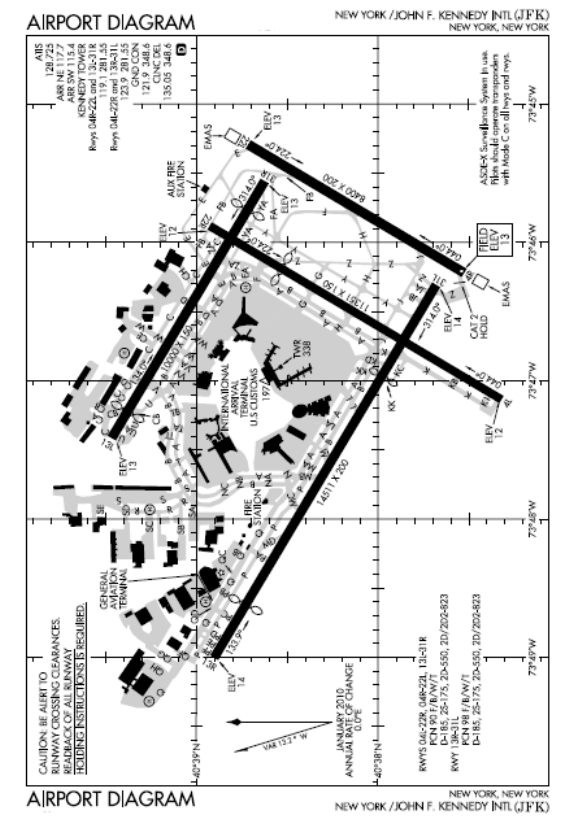

Figure 8. Airport diagram of John F. Kennedy International Airport (KJFK)

The actual runway configuration changed from $13 \mathrm{~L}, 22 \mathrm{~L} \mid 13 \mathrm{R}$ to $4 \mathrm{R} \mid 4 \mathrm{~L}, 31 \mathrm{~L}$ at $3: 00 \mathrm{PM}^{*}$. The TRCM algorithm selected the same initial and second runway configurations and advised the change to be made at 2:51 PM. This example supports that controllers are currently able to select runway configurations and TRCM is able to replicate these selections. In addition, TRCM planned which flights would be the last to use the first configuration and first to use the new configuration.

TRCM does not always select the same runway configurations as were historically used. On June 24, 2009, according to Aviation System Performance Metrics (ASPM) data, KJFK changed from runway configuration 22L, $22 \mathrm{R} \mid 22 \mathrm{R}$ to $22 \mathrm{~L} \mid 22 \mathrm{R}, 31 \mathrm{~L}$ at 3:00 PM as demand shifted from heavier arrivals to heavier departures. For this traffic scenario, TRCM was seeded with the actual initial configuration. TRCM advised changing the configuration

${ }^{*}$ The data source used to provide the actual runway configuration was limited to 15 -minute resolution.

14

American Institute of Aeronautics and Astronautics 
to $13 \mathrm{~L}, 22 \mathrm{~L} \mid 13 \mathrm{R}$ immediately and then to $4 \mathrm{R} \mid 4 \mathrm{~L}, 31 \mathrm{~L}$ at $3: 37 \mathrm{PM}$. Both runway configuration schedules were simulated for 79 flights between 3:00 and 4:00 PM and metrics compared. The TRCM configuration plan resulted in 245 minutes of total delay, as opposed to 360 minutes of delay for the runway configurations actually used. TRCM's initial configuration change provides independent arrival and departure runways, rather than a mixed-use runway. The later change to a configuration that favors departures reduced arrival delays before departure queues started to form. This example, while suggesting that planning runway configurations and change times may provide benefit, suggests that shadow-mode testing is needed to discuss with controllers why they would make certain decisions, possibly leading to enhancement to TRCM to ensure operational acceptance.

\section{Benefits}

Expected benefits for SORM include supporting traffic growth, cost reduction as a result of system efficiency, NAS optimization from metroplex operations, fairness in aircraft operations, and rational decision making. SORM's two primary elements, RCM and CADRS, are distinct technologies that individually will enhance airport performance, but they will perform best in unison.

Airport capacity for SORM increases were estimated during each 15 minute window during the year 2009 for each of the 77 airports in the FAA's ASPM database, depending on the runway configuration used at the time. Out of this massive data collection and analysis, a simple measure of the capacity benefit was the change in the average airport capacities, measured by Airport Arrival Rate (AAR) and Airport Departure Rate (ADR). Results indicated that the average AAR and ADR increased from 47.9 to $53.3 \mathrm{flight} / \mathrm{hr}$. (11.3\%) and from 45.7 to $46.6 \mathrm{flight} / \mathrm{hr}$. $(1.9 \%)$, respectively.

The throughput benefit estimate employed a methodology in which the benefit was defined as the difference between the number of scheduled flights with and without SORM. Using eight sample days, the unconstrained annualized airport operations (demand) at ASPM 77 airports in 2018 and 2025 were estimated to be 23.52 and 27.16 million, respectively. Without SORM, the projected throughput in 2018 and 2025 was 22.27 and 24.64 million operations, respectively. With SORM, the projected throughput in 2018 and 2025 was 22.44 and 24.90 million operations, respectively. In other words, SORM would enable an extra 170,000 and 270,000 annual operations in 2018 and 2025 , representing roughly $0.7 \%$ and $1 \%$ of the unconstrained operations in those years, respectively.

\section{Summary}

The runway is a constraining factor to operations in the NAS. NASA developed the SORM concept to address future runway management capabilities. The SORM concept addresses single- and metroplex-oriented runway management while considering TFM requirements. To date, the SORM accomplishments include: an assessment of current operations, algorithms to address single-airport operations, systems analysis to develop a methodology for selecting runway configurations in a the metroplex and a benefits assessment. The initial set of algorithms to support single airport operations are complete and work on the initial algorithm(s) for metroplex operations is currently underway and expected to be completed in May, 2012. A benefit assessment of SORM capabilities would enable an increase of roughly $0.7 \%$ in 2018 and $1 \%$ in 2025 .

The Federal Aviation Administration (FAA) and NASA have established four Research Transition Teams (RTTs) to identify concepts for transition to the FAA for NextGen. Under the Integrated Arrival/Departure/Surface (IADS) RTT, RCM has been identified as a Research Transition Product for both the single airport as well as the metroplex RCM capability. Delivery of the final products to the FAA is slated for Fiscal Year (FY) 2012 for single airport and FY 2014 for metroplex.

\section{Future Work}

The SORM work is building on the foundation based on an understanding of current and envisioned runway management capabilities for the future. The current algorithm supporting single-airport operations calculates an airport operating point and determines the best configuration based on weather and traffic demand. Planned enhancements include user preferences, airport operator constraints, consideration of revised wake vortex separation standards and TFM constraints. Current efforts under SORM are focused on developing runway management capabilities for the metroplex environment. Integration of SORM with concepts addressing surface and airspace operations is underway. Work within the next year also includes development of a user interface. The evaluations conducted for aggregate taxing strategies yielded promising results, however, shadow-mode testing would permit a 
dialogue with air traffic personnel to discuss the rationale for certain decisions. Finally, as progress is made in other research areas such as surface and airspace operations and TFM, SORM will continue to adapt its capabilities to ensure maximum effectiveness of intended functions.

\section{Acknowledgements}

The authors of this Paper acknowledge with gratitude, the contributions of the following individuals:

LETech - John Foggia

LMI - George Hart, and Carol DeZwarte

\section{References}

1. Joint Project Planning and Development Office, "Concept of Operations for the Next Generation Air Transportation System”, Version 2.0, Jun 132007.

2. Joint Project Planning and Development Office, "Research Activities [IWP2013]", URL: http://jpe.jpdo.gov/ee/request/filter?id=32903.

3. Lohr, G. W. and Williams, D. M. "Current practices in runway configuration management (RCM) and arrrival/departure runway balancing," NASA TM-2008-215557, 2008.

4. Federal Avaiation Admistration, "Air Traffic Control”, FAA Order 7110.65T, February 2010.

5. Federal Avaiation Admistration, "National Safety and Operational Criteria for Runway Use Programs", FAA Order 8400.9, November 1981.

6. Bott, T. F., Eisenhawer, S. W. and Foggia, J. R., "Systems Modeling of a Combined Arrival/Departure Runway Scheduling Decision Support Tool for System Oriented Runway Management", NASA Contractor Report, 2009.

7. Eisenhawer, S. W. , Bott, T. F., and Foggia, J. R., "Systems Modeling of a Runway Configuration Management Decision Support Tool for System Oriented Runway Management", NASA Contractor Report, 2009.

8. Lohr, G. W., Brown, S., Stough III, H. P., Atkins, S. A., Eisenhawer, S. W., Long, D., "System Oriented Runway Management: A Research Update", Ninth USA/Europe ATM Research and Develepment Seminar, 2011

9. Eisenhawer, S. W. , Bott, T. F., and Foggia, J. R., "System Model for Runway Configuration Management in a Metroplex Environment", NASA Contractor Report, 2011.

10. Weld, C., Duarte, M., Kincaid, R., "A Runway Management Model with Marginally Decreasing Transition Capabilites", Hindawi Publishing Corporation, Volume 2010, 2010.

11. Provan, C.A. and Atkins, S. C., "Tactical Runway Configuration Managment", Integrated Communications Navigation and Surveillance (ICNS) Conference, 2011. 